\title{
Les TIC dans l'entreprise : entre urgence et réactivité
}

\section{Théodora Pélage Mière}

\section{(2) OpenEdition}

1 Journals

Édition électronique

URL : http://journals.openedition.org/communicationorganisation/3410

DOI : 10.4000/communicationorganisation.3410

ISSN : $1775-3546$

Éditeur

Presses universitaires de Bordeaux

Édition imprimée

Date de publication : 1 juin 2006

Pagination : 196-205

ISSN : 1168-5549

\section{Référence électronique}

Théodora Pélage Mière, "Les TIC dans l'entreprise : entre urgence et réactivité », Communication et organisation [En ligne], 29 | 2006, mis en ligne le 21 juin 2012, consulté le 18 décembre 2020. URL: http://journals.openedition.org/communicationorganisation/3410; DOI : https://doi.org/10.4000/ communicationorganisation.3410

Ce document a été généré automatiquement le 18 décembre 2020.

(C) Presses universitaires de Bordeaux 


\title{
Les TIC dans l'entreprise : entre urgence et réactivité
}

\author{
Théodora Pélage Mière
}

\section{La question de l'urgence dans l'entreprise}

1 La question de l'urgence nous a paru intéressante à analyser dans deux cas : les services commerciaux de deux opérateurs de téléphonie mobile et dans la gestion des projets complexes (mécatronique). Cette urgence se définit de plus en plus comme l'obligation de livrer dans des délais très courts des produits ou services demandés par les clients. En ce sens, c'est toute la communication qui va se trouver au cœur de cette nouvelle exigence auxquelles les grandes organisations doivent faire face. Dans cette nouvelle configuration, les TIC Intranet et autres dispositifs deviennent des outils de gestion de l'urgence " pour donner la bonne information à la bonne personne » (Gramaccia, 2004, p. 24). Ce sont les messageries, les téléphones portables, les systèmes d'information qui fournissent des informations et des indicateurs en temps réel. Les technologies de l'information et de la communication ${ }^{1}$ sont souvent présentées par les acteurs de l'offre et les cadres dirigeants comme la condition d'une circulation rapide de l'information dans toute l'organisation, ce qui permettrait aux salariés d'être autonomes, réactifs et efficaces. Le gain de temps et de moyens est devenu des axiomes incontournables dans l'entreprise. La logique de court terme qui prévaut sur certains marchés concerne l'ensemble des entreprises et des secteurs jusque-là épargnés notamment les anciens monopoles de service public. Dans les secteurs tels que l'aéronautique civile, l'automobile, la téléphonie mobile, etc. (Sagem mobile \& Défense, Alcatel, Wanadoo, etc.), les salariés doivent être disponibles, réactifs, flexibles et s'adapter en permanence. Ils disposent pour certains de systèmes de production en temps réel et de systèmes d'information fournissant des indicateurs en temps réel. Le régime d'urgence est devenu le régime normal de fonctionnement dans les grandes entreprises soumises à une forte concurrence nationale et internationale exigeant d'être le premier à présenter une innovation dans un domaine particulier ou dans le cas où on est devancé par ses concurrents, mais surtout à répondre aux exigences du client qui change 
constamment d'orientation et de besoins. Ce client a d'ailleurs le choix devant une multitude d'offres.

2 L'entreprise est très sollicitée, il faut aller le plus vite possible et être très efficace pour devancer le concurrent mais aussi pour présenter un programme de développement et une qualité de production meilleurs que ceux du concurrent afin de gagner le marché et la confiance du client. Ce mode de fonctionnement inhabituel notamment auprès les salariés d'un ancien organisme de recherche public a nécessité la mise en place d'une stratégie de communication pour contribuer à une évolution des mentalités vers les nouvelles valeurs de l'entreprise et une réorganisation de la structure interne désormais tournée vers le client.

\section{Demande d'une plus grande réactivité : masse d'informations}

Des recherches sur l'introduction des TIC dans l'entreprise ont toutes montré les nouvelles dimensions dans la dynamique des échanges au sein des groupes notamment entre sites, unités, personnes éloignées géographiquement et contraintes d'échanger de nombreuses informations. Elles rendent possibles également la communication en temps réel entre différents acteurs impliqués dans une activité de projet séparés dans l'espace et parfois dans le temps (Benghozi, 2000 ; Gramaccia, 2004). Une étude sur les pratiques de travail des commerciaux chez un opérateur de téléphonie mobile permet de constater que ceux-ci se retrouvent avec une charge de travail conséquente du fait de la gestion de portefeuille clients mais également de la mise à jour via l'Intranet des produits, des tarifs et des spécifications. Dans le même temps la transmission régulière et souvent en temps réel des données du terrain pour permettre à la R\&D de proposer des produits encore plus adaptés, telles sont les exigences de l'entreprise auxquelles doivent se plier les commerciaux.

4 La situation actuelle de l'entreprise consiste à vouloir faire mieux que ses concurrents, à se tenir au courant de leurs innovations, à les suivre, voire à les copier. Ce qui se traduit dans l'entreprise par une pression plus forte envers l'encadrement pour être au courant des nouveautés et pour informer l'équipe de celles-ci. De même, les commerciaux doivent également s'informer des nouvelles technologies mises au point dans la fabrication des produits mais aussi des offres des concurrents pour pouvoir répondre aux questions des clients mieux informés qu'auparavant.

5 Chez les opérateurs de téléphonie mobile, en général ce sont les agents des services après-vente ou de la hotline qui doivent gérer un nombre important d'appels et répondre aux attentes des clients. Il est arrivé qu'ils apprennent en même temps que le public (par des spots publicitaires) le lancement d'un produit de leur entreprise. L'urgence et la réactivité sont les maîtres mots de la stratégie du top management et de la politique de communication qui l'explique et la justifie auprès des salariés. Exprimer ses doutes équivaut à ne pas être réactifs. On assiste dans l'entreprise à une triple temporalité entre la mise sur le marché du produit, le temps souvent négligé de la formation et de l'information des commerciaux et celui du centre opérationnel qui doit gérer au quotidien la relation avec les clients de plus en plus exigeants. Le quotidien du commercial nécessite désormais avant de pouvoir répondre aux demandes des clients, d'aller sur intranet ou sur des pages spécialisées pour s'informer des nouveautés et 
d'apprendre les argumentaires mis à sa disposition. D'ailleurs, le plus souvent, les commerciaux n'ont accès qu'aux sites fournissant des informations sur les produits, c'est-à-dire leur permettant de réaliser leurs tâches au quotidien. C'est le chef d'équipe qui souvent va sur certains sites et en tirent des informations dont il fait la synthèse à l'ensemble de son équipe. Les dernières informations sont envoyées par courrier électronique ou distribuées sur support papier.

6 C'est une masse d'informations importantes que le commercial de base doit connaitre, gérer, mémoriser au risque de passer pour un incompétent face au client qui est au bout du fil ou en face. C'est un travail qui demande du temps car tous les hommes ne sont pas égaux face à l'accès à l'information et le tri des données nécessite la mise en place d'une véritable stratégie de recherche (mots clés, pertinence, fiabilité et validité des informations). Le cadre qui gère une équipe de huit commerciaux doit en même temps se tenir au courant des nouveautés disponibles sur Intranet mais également lire les courriers électroniques. Toutes ces activités de lecture, de tri des informations demande du temps et est contradiction avec le gain de temps et la rapidité prônés par l'entreprise. Des efforts ont été réalisés du point de vue ergonomique pour rendre plus facile l'accès aux informations mises à disposition sur les sites Intranet et favoriser leur consultation.

7 Tout comme le changement, l'urgence fait partie des éléments du cadre dans lequel s'exercent les activités d'un grand nombre d'entreprises et constitue un élément important $\mathrm{du}$ « discours de conviction dogmatique » repris dans une large mesure dans les supports de communication interne. Ce discours présente comme inéluctable la gestion en urgence de certaines activités de l'entreprise pour faire face à la concurrence, gagner des parts de marché, satisfaire le client, etc. La réactivité, la maitrise de l'urgence sont présentées comme des réponses aux pressions externes dans le secteur privé comme dans le secteur public ayant connu un autre fonctionnement. Chaque salarié, quelque soit son statut, doit être capable de travailler dans un nouveau contexte, de s'adapter rapidement, d'abandonner une mission pour une autre, ceci à la demande du client qui fait vivre l'entreprise. Les ingénieurs en R\&D regrettent ce manque de temps qui leur permettrait de mieux gérer leur travail au quotidien. Pour certains, il faut livrer dans les meilleurs délais le produit ou service au client, faire face à la concurrence interne (entre laboratoire de recherche) mais également communiquer sur son travail, participer à des salons, à l'occasion répondre aux questions des journalistes et faisant visiter les locaux de l'institution aux acteurs clés de la région. En effet, l'innovation ou la fabrication des produits innovants est un des axes stratégiques et communicationnels de l'entreprise des secteurs de la téléphonie mobile, de l'automobile, qui lui permet de se démarquer de ses concurrents. Dans certains organismes publics de recherche, le choix de la recherche à court terme orientée vers les attentes des clients internes et externes a eu pour conséquence l'abandon de la recherche fondamentale et la concurrence entre les laboratoires.

8 Autre caractéristique de l'entreprise moderne, dans un contexte de plus en plus concurrentiel, la compétitivité est désormais liées à la capacité à maîtriser les échanges informationnels. Le marché mondial exige une plus grande efficacité, les entreprises sont confrontées à la globalisation de l'économie et à la gestion du changement. Les décisions doivent être prises rapidement, la fabrication de produits de meilleure qualité tout en restant concurrentiels sur les tarifs, dans ce contexte, les TIC ont leur rôle à jouer. 


\section{Les TIC : Réponse à une maîtrise de l'urgence?}

Notre expérience sur le terrain nous a permis de constater que les solutions aux problèmes de communication sont souvent présentées sous forme d'outils qui permettraient d'améliorer la communication dans les organisations. C'est le cas des opérateurs de téléphonie mobile qui accordent désormais beaucoup d'importance à la mise à jour du site des commerciaux pour permettre de faire face à des nouveautés au jour le jour. Les salariés doivent répondre dans les brefs délais aux questions des clients de plus en plus exigeants et informés. Il peut s'agir d'une nouvelle technologie mise en place sur le marché sur laquelle on attend du commercial qu'il soit déjà au courant et qu'il ait une parfaite maîtrise de son utilisation.

Actuellement, nous travaillons sur la communication dans la gestion des projets mécatroniques dans lesquels interagissent mécaniciens, électroniciens et informaticiens en collaboration dans le secteur automobile avec pour interrogation principale comment faire interagir ces trois acteurs aux contraintes et aux intérêts différents tout en respectant les délais et en répondant aux besoins des clients sans cesse fluctuants? Pour assurer la gestion des projets industriels, l'entreprise est souvent constituée d'une partie R\&D, composée d'un responsable de projet, d'ingénieurs, de techniciens, de spécialistes, de sous-traitants, etc. Une autre branche comprend un responsable ligne de produits, avec un responsable programmes, des ingénieurs qualités, des ingénieurs sécurités, des commerciaux, etc. Chaque unité développe en interne des microprojets en vue de finaliser un besoin client.

Dans la gestion des projets industriels, les TIC seraient une solution miracle pour faire face à l'imprévu et au respect des calendriers. Pour suivre l'évolution du projet, chaque intervenant doit mettre à jour une base de données commune selon des standards de présentation prenant en compte les éventuelles modifications sur le développement de produit. Le chef de projet est au centre de l'urgence car il doit répondre aux besoins techniques du client en respectant le planning et en limitant le plus que possible les ressources allouées en interne. De ce fait, il est quotidiennement en interaction avec ses ressources techniques mais surtout avec la partie programme dont le rôle consiste à veiller au respect des jalons et à allouer certaines ressources en cas de nécessité. La branche programme est en relation directe avec le client, elle propose une réponse technico-commerciale à l'appel d'offre et impose une planification des tâches et des jalons au responsable de projet (RdP). Entre l'ingénieur R\&D et le client, il y a donc tout un ensemble d'acteurs ayant chacun ses propres centres d'intérêt.

12 L'urgence prend forme à partir du moment où les spécifications (techniques et plannings) du client changent d'orientations. L'implication directe se fait sentir au niveau du RdP par de nouveaux développements qui introduisent du coup de nouvelles qualifications. Il faut donc disposer d'un champ d'expériences dans le domaine en question afin de pouvoir prévenir ces changements, mais aussi d'une équipe pluridisciplinaire et de qualité qui soit capable d'identifier très rapidement les implications que ces nouveaux besoins peuvent engendrer. Une bonne analyse et une compréhension des informations relatives aux standards internationaux et des technologies mises en oeuvre sont les solutions simples et efficaces pour répondre à ces urgences, qui le plus souvent sont des besoins non réalisables dans les délais imposés et peuvent dépasser la technologie existante. La disponibilité de l'information et une 
bonne expérience du domaine sont par conséquent des atouts importants pour répondre à ces urgences, car ne l'oublions pas, il faut réaliser urgemment avec efficacité un produit qui le plus souvent répond à des normes internationales drastiques où la sécurité de l'utilisateur est primordiale. Régulièrement en interne des rencontres sous forme de réunions sont proposées par le responsable du projet pour faire un état de lieux des actions en cours et surtout de distribuer des priorités (donc l'urgence) à certaines tâches. Des signaux sont régulièrement émis via les réseaux internes (Intranet, réseaux FTP, messagerie électronique, etc.) par la plateforme qualité afin d'identifier les urgences et de mettre en conséquence les actions nécessaires. Dans cette configuration, les enjeux communicationnels vont au de-là d'une simple coordination des projets ou d'un travail en réseau. En fait, chaque ingénieur doit redéfinir sa manière de travailler, de regarder son activité, d'en rendre compte et d'intervenir sur celles des autres. Ce qui exige du temps surtout lorsque le quotidien ressemble à une gestion des urgences.

Les acteurs sont constamment dans un contexte d'urgence, car les étapes du cycle en $\mathrm{V}$ $\mathrm{du}$ développement initialement prédéfinies sont modifiées tout au long du design, causé par la superposition au besoin initial de nouvelles spécifications. La moindre modification du besoin équivaut à une mise à jour du dossier de définition et à une vérification par rapport aux matrices de conformité, image directe du besoin initial. Ces documents demandent des accords internes, des validations, des discussions techniques, administratives. Les responsabilités du chef de projet, de l'ingénieur, de l'équipe qualité peuvent être engagées, ce qui peut être source de blocage. Lorsque l'information n'est pas clairement définie, elle ne peut être véhiculée, ce qui peut entraîner un ralentissement du projet surtout si les structures qui interagissent dans un projet (qualité, sécurité, programme, URD, etc.) sont lourdes et ne permettent pas une meilleure circulation de l'information partant du fournisseur de service vers le client. Pour pallier à ces incertitudes et minimiser les répercussions, des outils ont été mis en place et des formations associées à ces nouveaux moyens. Ces outils permettent une interaction entre différents équipes et notamment, du client vers l'Unité de Recherche et de Développement ${ }^{2}$, de l'URD vers ses ressources (sous-traitants, ingénieurs, techniciens, service commercial, etc.). Ces moyens se présentent sous la forme de dossiers officiels qui reprennent les problèmes ainsi que les solutions proposées et les contraintes engendrées. La circulation de ces données est assurée par des systèmes informatiques sécurisés utilisant comme support les réseaux interentreprises, la messagerie, les bases de données intelligentes, etc. L'objectif est de faire réagir rapidement le noyau concerné afin de limiter le plus que possible le glissement des jalons. La mise en place de ces éléments suppose une structuration de l'entreprise en adéquation avec les nouveaux moyens et les nouveaux besoins du client pour faciliter la circulation bidirectionnelle des données et la prise en compte des flux des informations de manière à répondre le plus rapidement aux demandes versatiles du client.

Le chef de projet peut ainsi faire des suggestions, des propositions, informer son client, ainsi que son responsable programme. Attirer l'attention très vite, officialiser les actions lancées, valider, tracer les différences, les modifications tout au long du projet, tels sont les points essentiels que constitue la réactivité dans un contexte d'urgence dans une entreprise évoluant dans un secteur sensible (aéronautique, mobile, etc.), le but ultime est de limiter les débordements par rapport aux jalons prédéfinis initialement. Dans un contexte où interagissent plusieurs types de métiers, « tout va 
très vite ", le client demande souvent la livraison de son produit dans des délais très courts.

Autre configuration, lorsque le produit d'un concurrent rencontre du succès, l'industriel doit lancer dans les plus brefs délais, un produit ayant la même technologie ou équivalente, pour ne pas être en reste de la course à la rentabilité. Les différentes publicités des fournisseurs d'accès à Internet (Free et la freebox et Wanadoo et la livebox) nous montrent à quel point ceci est une réalité dans les activités quotidiennes des ingénieurs et des commerciaux. Ainsi pour faire face à l'urgence de type concurrentiel, certaines URD ont tendance à négliger certains éléments du cycle de développement du produit non traités avec autant de rigueur qu'en temps normal (modification du plan de qualification, de validation, des tests recette, etc.). En 2005, un fournisseur d'accès à Internet avait été obligé de rappeler un produit défectueux mis sur le marché pour rivaliser un concurrent. Dans la course à la compétitivité, les dirigeants sollicitent les ingénieurs qui doivent développer dans de brefs délais pour faire face à la concurrence. L'analyse des causes ayant pu entraîner le crash d'un avion de la compagnie aérienne britannique British Airways (Vol BA 5390 du 10 juin 1990) avait montré que l'ingénieur n'avait pas choisi les bons boulons pour fixer le pare-brise, pressé par le temps, il avait dû faire confiance à son expérience visuelle et donc ne pas consulter les procédures et les spécifications. L'enquête avait cependant déterminé que l'accident relevait d'un problème plus général lié à l'organisation interne de la société de service. Cet accident n'avait heureusement pas fait de victimes, ce qui n'est pas toujours le cas. La non-maîtrise de l'urgence et une organisation non adaptée peuvent engendrer dans certains cas des conséquences graves.

16 Les industriels suivent un cycle en $\mathrm{V}$, qui est fonction de l'industriel et dépend du contexte (civil, militaire, etc.) avec des contraintes lourdes à gérer (les différentes phases) indispensables pour garantir la sécurité du projet et être en conformité avec les normes internationales. Le client a souvent des besoins urgents et fluctuants dans le temps, le chef de projet doit avoir un regard particulier et se mettre à la place du client, anticiper ses attentes, prévenir certains changements de cap, donc mener en permanence une réflexion à large vision. Dans une petite structure, il faut être polyvalent, flexible et réactif. Le chef de projet doit être expérimenté dans son domaine d'expertise (certification, robustesse, qualification du produit, validation, etc.) mais aussi posséder une vision pluridisciplinaire, être au courant de l'avancée d'autres projets dans l'entreprise afin de tirer profit des analyses de situation. Il doit également faire passer l'information vers les ingénieurs et ses ressources techniques. Une communication transmise à temps et à la bonne personne qui doit être réactive est au cœur de ce processus.

17 Le chef de projet mécatronique par exemple est à la tête d'une équipe pluridisciplinaire au sein de laquelle on trouve des électroniciens, des informaticiens, des mécaniciens, etc. Avec la concurrence internationale, les demandes des clients deviennent importantes et nécessitent une haute technicité. Dans la téléphonie mobile, la production se chiffre en million d'unités, il faut être sûr de la spécification et de la définition du produit, ce qui est encore source de pression sur le travail. Les qualités du produit évoluent sans cesse ; en puissance, en définition, en poids, en autonomie, en taille (ergonomie), avec des fonctions de plus en plus nombreuses, complexes et perfectionnées. Tout est miniaturisé, il faut répondre au client qui veut tout avoir dans un volume restreint et pour un moindre coût. 

dans un contexte de concurrence farouche avec les technologies des pays émergents (téléphonie, vidéo, automobile, etc.). Ces nouveaux marchés imposent des volumes importants avec des composants de très bonne qualité pour un coût de production moindre. Le chef de projet doit donc disposer d'outils qui lui permettent d'avoir une meilleure visibilité des tâches, des activités de chacun pour une meilleure optimisation de la gestion du projet. La formation à l'utilisation de certains outils aidant à la gestion de projets (MS project,...) est mise en place. La conduite du projet est devenue un module important dans la formation des futurs ingénieurs avec un intérêt croissant sur une bonne répartition des tâches et les modalités de communication précises tout au long du projet. La planification est au cœur du management du projet. Il s'agit de mieux définir les actions à réaliser, de fixer des objectifs, de coordonner les diverses actions, ce qui nécessite au préalable une vision claire et précise du projet et des qualités de manager car l'usage d'un outil ne suffit à lui seul à gérer les problèmes liés à l'urgence. Il y a sans cesse des aléas auxquels l'équipe doit faire face. Ces outils ne montrent leur efficacité que s'ils rencontrent l'adhésion de tous les acteurs du projet qui une fois formés participeront de manière régulière à son fonctionnement.

\section{Conclusion}

Cette étude montre la communication des organisations s'adapte toujours autant et de manière très importante à la stratégie des entreprises. Celles-ci attendent toujours des outils miracles pour atteindre leurs objectifs et faire face aux contraintes de leur environnement. Dans la gestion de projets complexes, la transmission des informations en temps réel et surtout à la bonne personne permet de réagir au plus vite à la demande des clients et de ce fait, sa gestion quotidienne devient un élément de fonctionnement des entreprises. Les outils modernes de communication servent de prétextes pour justifier l'urgence et la rendre régulière, puisque ceux-ci permettraient de faire circuler l'information et de gérer les aléas que les dirigeants refusent de prendre en compte, leurs préoccupations étant essentiellement axées sur des questions de rentabilité.

\section{BIBLIOGRAPHIE}

Benghozi PJ, d'Iribane A., Flichy P., (coordonné par), « Internet en entreprise », in Réseaux, n 104, Vol 18, 2000.

Germain M., L'intranet, Paris, Ed. Economica, 1998.

Gollac M., «Le capital est dans le réseau. La coopération dans l'usage de l'informatique », in Travail et emploi, $\mathrm{n}^{\circ}$ 3/96, pp. 39-60

Gramaccia G., Miny M., Vezie G., La communication dans les projets, AFNOR, 2004.

Communication et organisation, 29 | 2006 
Guyot B., « Mettre en ordre les activités d'information, nouvelle forme de rationalisation organisationnelle », novembre 2002, texte disponible sur le site http://www.u-grenoble3.fr/ les_enjeux.

Lépine V., « Les enjeux communicationnels et socio-organisationnels du déploiement de dispositifs de groupware en entreprise ", Thèse soutenue à l'université Stendhal-Grenoble 3, 2000.

Lépine V., « Une entreprise sidérurgique aux prises avec le groupware : apports d'une étude empirique ", novembre 2002, texte disponible sur le site http://www.u-grenoble3.fr/les_enjeux..

Martin F., « Réunions décisionnelles médiatisées par ordinateur en entreprise », Thèse soutenue à l'université Lyon 2, 1997.

Vacher B., La gestion de l'information dans l'entreprise, ADBS, 1997

\section{NOTES}

1. Désormais TIC

2. Désormais URD, Unité de Recherche \& de Développement dans les entreprises de logiciel et de téléphonie mobile.

\section{RÉSUMÉS}

La question de l'urgence ou sa gestion est actuellement au cœur des discussions des salariés. En effet, ceux-ci disent désormais devoir gérer les priorités de l'entreprise et les mettre en pratique au jour le jour. Face à cette situation, les dirigeants estiment que les outils modernes de la communication permettraient aux salariés de réagir au plus vite dans un contexte de grande concurrence. Cette étude a pour objet de présenter des éléments de réflexion sur la place des dispositifs de communication dans la gestion de l'urgence à partir du constat réalisé dans trois entreprises.

The question of urgency or of its management is a subject which is currently at the heart of discussions among employees. Indeed, these people say that, nowadays, they have to deal with company priorities and to put them into practice on a daily basis. Company managers, when faced with such a situation, believe that modern communication tools could enable their employees to react promptly in a context of strong competition. The aim of this project is to present a set of relevant elements for a discussion about the role of different means of communication in a context of urgency management, with examples taken from three different firms".

INDEX

Mots-clés : TIC, management de projet, urgence 


\section{AUTEUR}

\section{THÉODORA PÉLAGE MIÈRE}

Théodora Pélage Mière est Maître de Conférences à l'Université de Versailles Saint Quentin en Yvelines. Chercheur au LAREQUOI, Laboratoire en Management, son domaine de recherche est la communication des organisations. Elle s'intéresse à la place des dispositifs de communication dans le management des projets complexes notamment dans le secteur automobile. Mail : pelage.theodora@iut-mantes.uvsq.fr 